\title{
Fragilariaceae (Bacillariophyta) en humedales de ALtura de Catamarca (Argentina)
}

\author{
CLAUDIA T. SEELIGMANN ${ }^{1 *}$, NORA I. MAIDANA ${ }^{2-3}$ y EDUARDO A. MORALES ${ }^{4}$
}

\begin{abstract}
Summary: Fragilariaceae (Bacillariophyta) in high altitude wetlands of Catamarca (Argentina). Three high mountain wetlands were studied in the Catamarca Province, Argentina, as part of a larger study focusing on high mountain waterbodies in the Argentinian Andes. In this contribution we present species in the group of small Fragilariaceae found so far in: Laguna Diamante (hiperhaline), Laguna Salitre (fresh) and Laguna Negra (mesohaline). The species belong to the genera Staurosira, Staurosirella and Pseudostaurosira. Three species, Staurosirella galanensis, S. salitrensis and Pseudostaurosira ferrarioae are new to science, which are herein described, illustrated and compared to morphologically similar taxa. S. galanensis is smaller and more rhomboidal than the other cruciform species of the genus, while S. salitrensis is smaller and less silicified than closely related species. Pseudostaurosira ferrarioae is distinguished from similar taxa especially by its well developed apical pore fields. The other representatives of the family found in these sites were Staurosira binodis, which is a new record for the Catamarca Province, Pseudostaurosira altiplanensis, cited here for the first time for Argentina and $P$. aff. polonica.
\end{abstract}

Key words: Andes, fragilarioids, Bacillariophyta, diatoms, high mountain ecosystems.

\begin{abstract}
Resumen: Se estudiaron tres humedales de alta montaña en la provincia de Catamarca, Argentina, como parte de un estudio más amplio que se enfoca en cuerpos de agua en los Andes argentinos. En esta contribución damos a conocer dentro del grupo de Fragilariaceae de pequeño tamaño, especies de los géneros Staurosira, Staurosirella y Pseudostaurosira halladas en las lagunas Diamante (hiperhalina) y Salitre (dulce) y en la vega de la laguna Negra (mesohalina). De las especies encontradas, Staurosirella galanensis, S. salitrensis y Pseudostaurosira ferrarioae son nuevas para la ciencia y fueron descritas, ilustradas y comparadas con taxones similares. S. galanensis es más pequeña y romboidal que otras especies cruciformes del género, mientras que $S$. salitrensis es de menor tamaño y menos silicificada que otras especies estrechamente relacionadas. Pseudostaurosira ferrarioae se distingue de especies afines especialmente por tener campos de poros apicales bien desarrollados. Los otros representantes de esta familia encontrados en el área de estudio fueron Staurosira binodis, especie que se menciona por primera vez para Catamarca, Pseudostaurosira altiplanensis, citada por primera vez para Argentina y $P$. aff. polonica.
\end{abstract}

Palabras clave: Andes, fragilarioides, Bacillariophyta, diatomeas, ecosistemas de alta montaña.

\section{INTRODUCCIÓN}

En Argentina, los numerosos y variados cuerpos de agua en alta montaña (ubicados a más de $2000 \mathrm{~m} \mathrm{snm}$ ), se encuentran, según el esquema de Morrone (2002), dentro de las provincias Puna y
Prepuna. Estas provincias, biogeográficamente muy relacionadas, pertenecen a la Subregión PáramoPuneña. En el área de estudio existen numerosos humedales entre los que se encuentran lagos y salares de diversos tamaños, integrados en sistemas de cuencas endorreicas, que constituyen parches

${ }^{1}$ Universidad Nacional de Tucumán, Facultad de Ciencias Naturales e IML, Argentina.

2 Universidad de Buenos Aires, Facultad de Ciencias Exactas y Naturales, Departamento de Biodiversidad y Biología Experimental, Laboratorio de Diatomeas Continentales, Buenos Aires, Argentina.

${ }^{3}$ CONICET- Universidad de Buenos Aires, Instituto de Biodiversidad y Biología Experimental y Aplicada - (IBBEA), Laboratorio de Diatomeas Continentales, Buenos Aires, Argentina.

${ }^{4}$ Universidade de Évora, Laboratório da Água e Instituto Ciências da Terra, Évora, Portugal.

*claudiaseeligmann@gmail.com 
de hábitats acuáticos en una matriz desértica. Estos ecosistemas albergan una biodiversidad peculiar con especies endémicas, de las cuales las aves son especialmente variadas. Los lagos son poco profundos y no presentan una verdadera comunidad de plancton; hay una proliferación frecuente de formas adaptadas a baja humedad. Las diatomeas han sido reconocidas como el grupo de algas más importante, tanto en abundancia como en riqueza de especies, seguido de cianobacterias y clorofitas (Maidana \& Seeligmann, 2014). Los humedales son muy variables espacial y temporalmente y tienen una alta fragilidad debido, en parte, a las prolongadas sequías, alta irradiación, fuertes vientos y amplitudes térmicas elevadas (Caziani \& Derlindati, 2000).

Una gran parte de la diatomoflora andina es aún poco conocida y el número de endemismos y de nuevas especies podría ser mucho mayor que lo reportado hasta ahora en la literatura. A pesar de ello, se han descripto numerosos taxones, y se ha registrado un alto número de especies en algunos sitios de los Andes (Maidana \& Seeligmann, 2006, 2015; Maidana et al., 2011; Morales et al., 2009, 2014a; Rumrich et al., 2000; Seeligmann \& Maidana, 2003; Seeligmann et al., 2008, entre otros).

Durante las últimas tres décadas, los taxónomos prestaron especial interés a las especies de fragilarioides de pequeño tamaño. Estudios de la ultraestructura de sus valvas dieron como resultado, por ejemplo, la reasignación de varias especies tradicionalmente incluidas en Fragilaria Lyngbye en algunos géneros nuevos y otros reestablecidos a tal fin (Williams \& Round, 1987; Morales, 2001, 2002, entre otros). Entre los caracteres ultraestructurales más destacados actualmente utilizados para circunscribir los géneros se encuentran: la estructura de las aréolas, de las espinas y de los campos porosos apicales (CPAs).

Los géneros más ampliamente distribuidos de las pequeñas fragilarioides son Staurosira Eherenberg, Staurosirella Williams \& Round y Pseudostaurosira Williams \& Round. Las especies de Staurosira se caracterizan por ser cruciformes, con estrías compuestas por aréolas alargadas en sentido apical, espinas huecas, CPA poco desarrollados y bandas cingulares abiertas. Forman siempre colonias, son epífitas y accidentalmente pueden ser planctónicas. Staurosirella tiene valvas lineales, cruciformes o elípticas; puede haber una depresión en uno de los ápices, el borde abvalvar es paralelo a la unión valva/ manto y las aréolas son como hendiduras. Los CPAs están bien desarrollados, las espinas son huecas o sólidas, las bandas cingulares pueden ser abiertas o cerradas y las fimbrias están bien desarrolladas. En Pseudostaurosira las valvas son lineales, elípticas o cruciformes. Este género está caracterizado por presentar estrías cortas, formadas por areolas grandes, tener volae bifurcadas y robustas, CPAs reducidos, espinas sólidas y con lígulas, areolas con alerones y el manto con placas. Habitualmente, se encuentran formando colonias adheridas al substrato y accidentalmente pueden ser planctónicas.

La clasificación de las pequeñas fragilarioides de agua dulce es una de las más complejas dentro de las diatomeas (Morales, 2002) por su pequeño tamaño y características valvares que se hallan bajo el límite de resolución del microscopio óptico sumado al reducido número de caracteres y la relativa falta de estudios monográficos que ilustren la variabilidad intra y extrapoblacional, aun de los taxa más comunes. Esta escasez de información se hace extensiva a Sudamérica donde la carencia es más notoria, especialmente para ambientes de alta montaña. A esto se superpone la deriva taxonómica, que ha llevado a la existencia de más de un concepto para un solo taxón (Morales et al., 2014a). Es por ello que, a pesar de la frecuente ocurrencia de este grupo, falta un análisis detallado que incorpore información tanto de microscopía óptica (MO) como de microscopía electrónica de barrido (MEB) (Morales, 2005) y permita resolver la taxonomía de muchas especies.

Durante el análisis de muestras colectadas dentro de los proyectos realizados (Ecología de humedales de Alta Montaña) en la provincia de Catamarca, se observaron varios taxa de fragilarioides (Seeligmann \& Maidana, 2003; Maidana \& Seeligmann, 2006). En este trabajo presentamos un estudio detallado sobre tres especies que tienen un conjunto de características que las distinguen de otros taxones del grupo descritos anteriormente. Además, ilustramos otras tres especies que si bien ya han sido descritas no fueron citadas para Argentina o no habían sido mencionadas anteriormente para Catamarca o en ambientes tan extremos.

\section{Materiales y Métodos}

En el marco del Censo Internacional de Aves Altoandinas del GCFA, realizado en enero de 2005, se obtuvieron muestras de agua con red de plancton 


\section{T. Seeligman et al. - Fragilariaceae en humedales de altura}

de $20 \mu \mathrm{m}$ de apertura de malla en la zona litoral de tres humedales de la Provincia de Catamarca situados entre los $26^{\circ} 02^{\prime} 08^{\prime \prime}$ y los $27^{\circ} 39^{\prime} 23^{\prime \prime} \mathrm{S}$, a más de $3600 \mathrm{~m}$ snm (Fig. 1). El material recolectado fue incorporado al Herbario (index herbariorum LIL) Colección Ficológica de la Fundación Miguel Lillo bajo los rótulos: laguna Diamante: ARGENTINA. Prov. de Catamarca: Dpto. Antofagasta de la Sierra, 20-01-2005, Seeligmann 26103 (LIL), hiperhalina; laguna Salitre: ARGENTINA. Prov. de Catamarca: Dpto. Antofagasta de la Sierra, 20-01-2005, Seeligmann 26105 (LIL), dulce y en la vega de la laguna Negra: ARGENTINA. Prov. de Catamarca: Dpto. Tinogasta, 22-012005, Seeligmann 26108 (LIL), mesohalina. La salinidad, la conductividad eléctrica, el oxígeno disuelto y la profundidad se midieron en el campo. Las variables físico-químicas para cada ecosistema se pueden encontrar en Maidana \& Seeligmann (2015, Tabla 1).

El tratamiento de las muestras fue realizado siguiendo la metodología estándar descrita en Battarbee (1986). Se usó Naphrax ${ }^{\circledR}$ como medio de montaje en preparados permanentes.

Las observaciones se realizaron usando un microscopio óptico binocular Reichert-Jung Polyvar equipado con objetivos PlanApo 100X, NA 1.32 y una cámara digital Canon EOS 600D.

Para la observación con MEB se utilizó un microscopio Carl Zeiss SUPRA $40(15 \mathrm{kV})$ del Centro de Microscopías Avanzadas (CMA), Facultad de Ciencias Exactas y Naturales (FCEyN), Universidad de Buenos Aires, Argentina.

Los especímenes fotografiados tanto con MO como con MEB fueron medidos usando el programa Zeiss Axiovision 4.8.2.

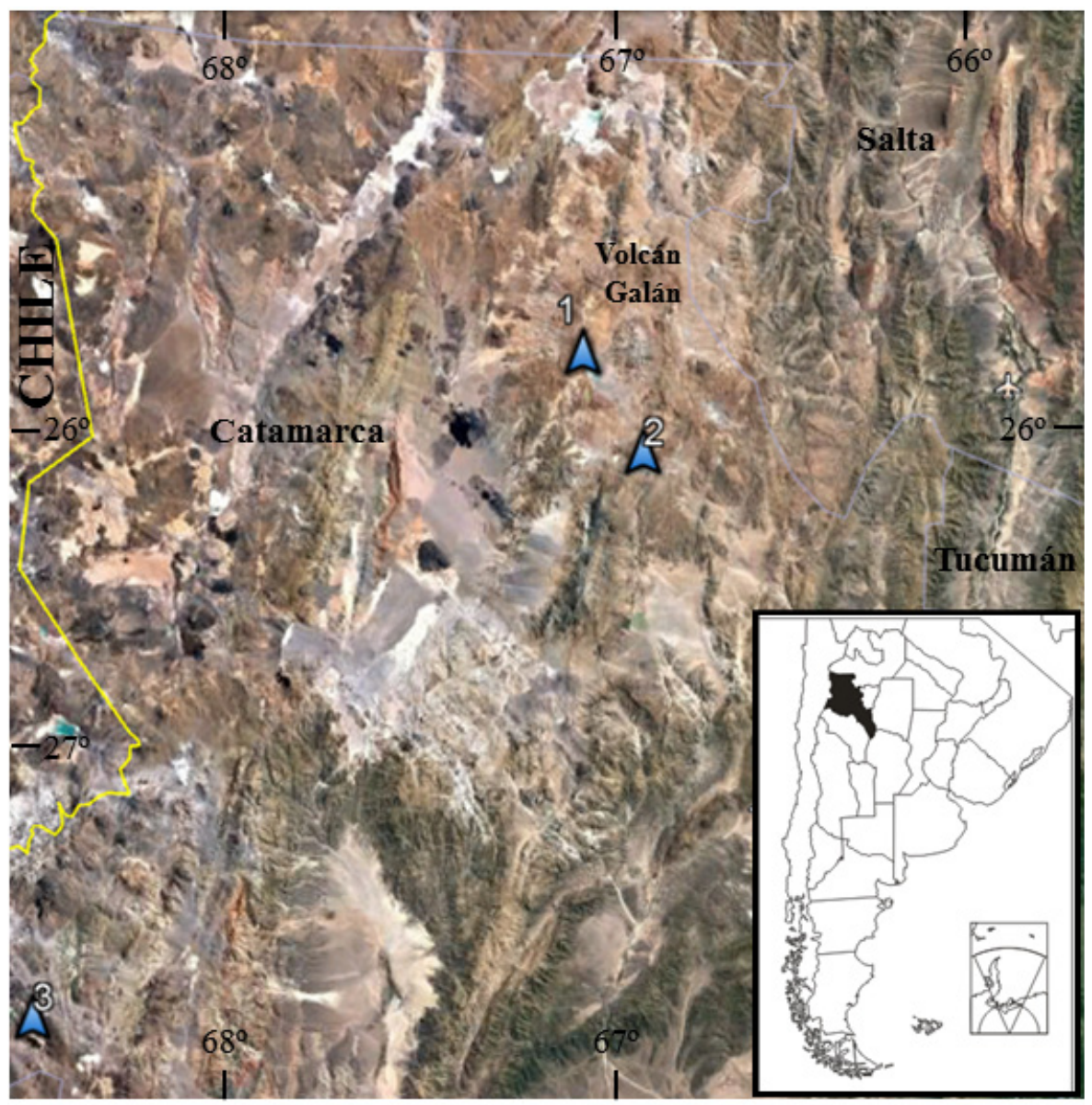

Fig. 1. Mapa del área de estudio: 1: Laguna Diamante, 2: Laguna Salitre, 3: Vega de Laguna Negra. 
Para la descripción de las nuevas especies se siguió la terminología sugerida por Ross et al. (1979) para la de estrías, areolas y espinas; la de Barber \& Haworth (1981) para la forma de la valva y patrón de estrías y la de Williams \& Round (1987) y Round et al. (1990) para la subestructura areolar, poros apicales y características de las bandas cingulares.

\section{Resultados y Discusión}

En los humedales ya estudiados para la provincia de Catamarca, hemos encontrado una notable riqueza de especies y una gran variabilidad morfológica de taxones pertenecientes a la familia Fragilariaceae (Maidana \& Seeligmann, 2015). Las lagunas con mayor número de fragilarioides fueron Salitre (10), Vega de la Laguna Negra (5) y la laguna Diamante (4).

En general, se considera que las pequeñas fragilarioides son formas habituales en ambientes con conductividades elevadas (Seddon et al., 2014) y Morales (2002) sostiene que son componentes importantes de la flora de aguas duras. Algunas especies de Pseudostaurosira fueron reportadas para ambientes con conductividad baja a media (CejudoFigueiras et al., 2011). Las Fragilariaceae en la Puna y Prepuna fueron halladas en un variado espectro de salinidad: en agua dulce (laguna Salitre), mesohalina (vega de la laguna Negra) e hiperhalina (laguna Diamante).

El análisis morfológico y morfométrico de las pequeñas Fragilariaceae halladas en las lagunas Diamante y Salitre y en la vega adyacente a la laguna Negra reveló la presencia de una sola especie del género Staurosira, S. binodis (Ehrenberg) LangeBertalot, que se menciona por primera vez para Catamarca, dos especies nuevas de Staurosirella: $S$. galanensis sp. nov. y $S$. salitrensis sp. nov. Asimismo, identificamos tres especies de Pseudostaurosira, de las cuales una representa una nueva especie para la que proponemos el nombre de $P$. ferrarioae sp. nov. Además, encontramos a $P$. altiplanensis LangeBertalot \& U. Rumrich, que se cita por primera vez para Argentina y a $P$. aff. polonica (M. Witak \& Lange-Bertalot) E. A. Morales \& M. B. Edlund.

Staurosira binodis (Ehrenberg) Lange-Bertalot (Fig. 2A-B)

Valvas lanceoladas en especímenes pequeños, algo constrictas en el centro en los más grandes,
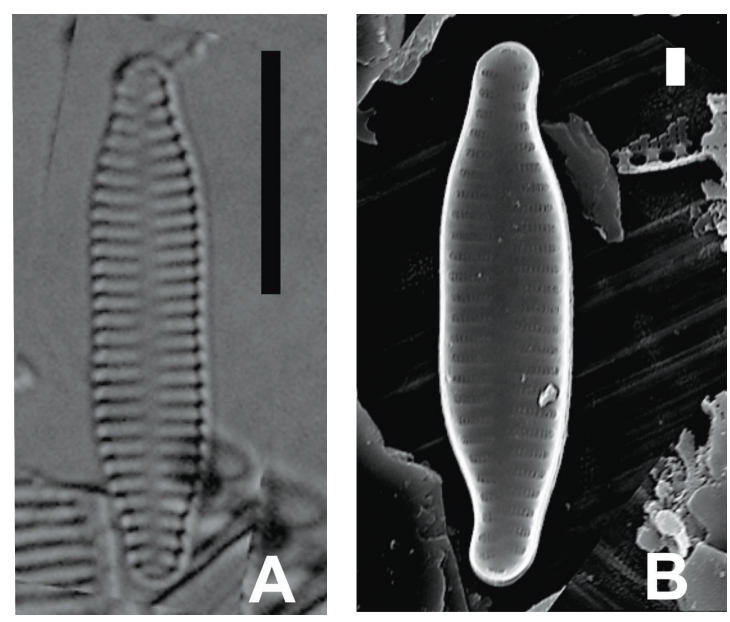

Fig. 2. Staurosira binodis. A: (MO). B: (MEB) vista interna. Escalas $=A$ : $10 \mu \mathrm{m}$; B: $2 \mu \mathrm{m}$.

con extremos subcapitados y ápices redondeados.

Dimensiones: eje apical 19,3-21,6 $\mu \mathrm{m}$, eje transapical 3,9-4,6 $\mu \mathrm{m}$. Estrías 13-14 en $10 \mu \mathrm{m}$

Material estudiado: laguna Diamante.

Staurosira binodis fue mencionada anteriormente para Argentina en Laguna de Los Pozuelos (Jujuy) como Staurosira construens var. binodis (Ehrenberg) Hamilton (González Achem et al., 2014).

Staurosirella galanensis Seeligmann, C. T., N. I. Maidana \& E. A. Morales sp. nov. (Fig. 3A-N)

Frústulos rectangulares en vista conectival. Valvas cruciformes, isopolares a levemente heteropolares. Extremos rostrados con ápices redondeados de longitud variable. Área axial angosta, lanceolada, delimitada por estrías levemente radiales. Las estrías son uniseriadas y están formadas por areolas alargadas en sentido apical, ininterrumpidas desde la superficie valvar hasta el manto (Fig. 3H-J). Las aréolas decrecen en tamaño desde el margen de la superficie valvar hacia el manto y hacia el área central. Espinas sólidas, marginales, ubicadas entre las estrías y en los polos (Fig. 3H, M), con ápices abruptamente espatulados (Fig. 3J, M). En las aréolas, las volae no fueron observadas $\mathrm{y}$, debido a la pequeña apertura areolar, es muy probable que estén muy reducidas (Fig. 3M, N). CPAs en ambos ápices, desigualmente desarrollados y pequeños, 
C. T. Seeligman et al. - Fragilariaceae en humedales de altura
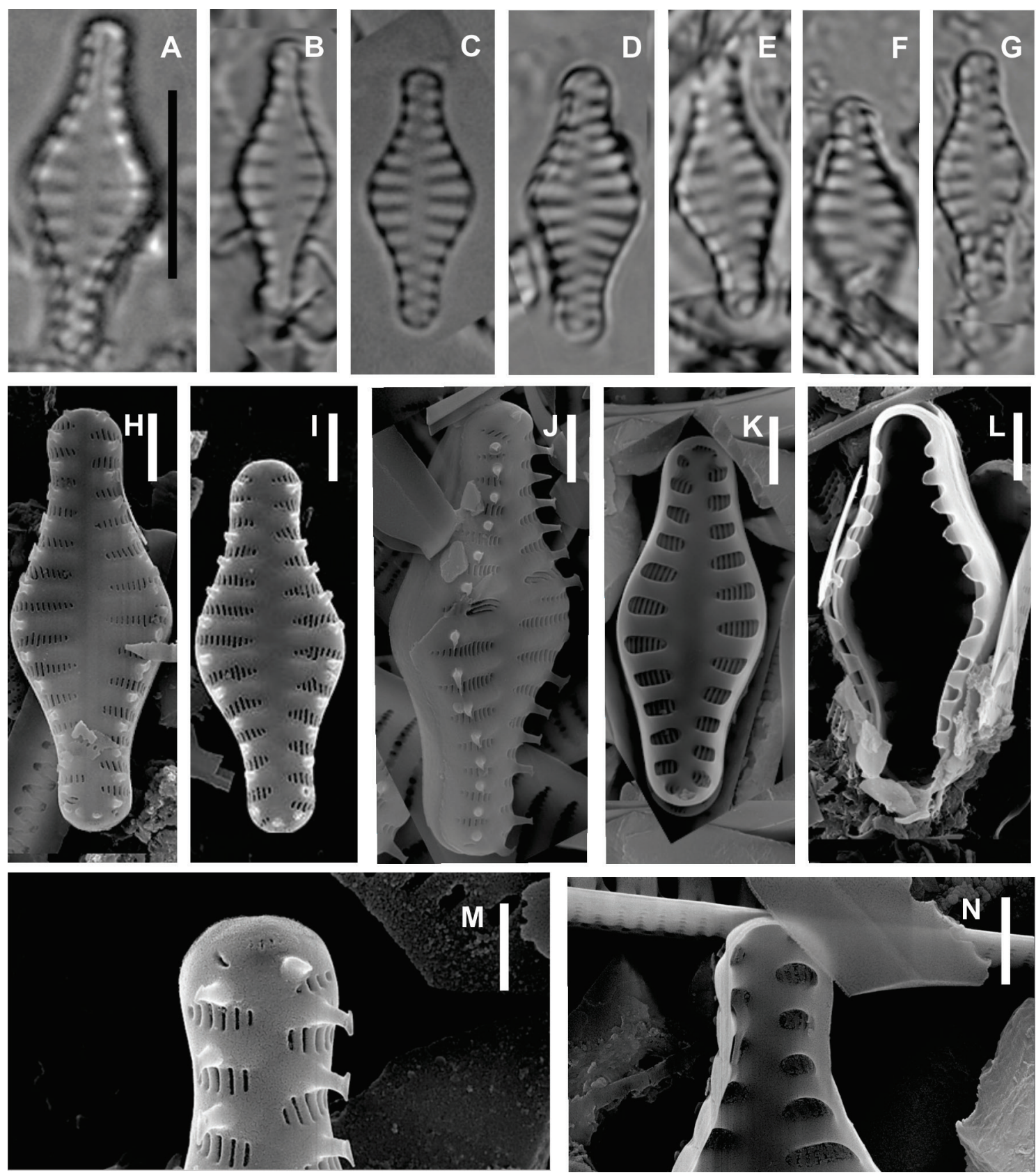

Fig. 3. Staurosirella galanensis. A-G: (MO). H-N: (MEB). H-J: vistas externas. J: par de estrías centrales con una posición anómala de las aréolas. $\mathbf{K}-\mathbf{L}, \mathbf{N}$ : vistas internas. L: valvocópula ancha y fimbrias bien desarrolladas. M: detalle de uno de los ápices mostrando un CPA formado por pequeños poroides y las espinas sólidas con extremos espatulados. Escalas = A-G: $10 \mu \mathrm{m} ; \mathrm{H}-\mathrm{L}: 2 \mu \mathrm{m} ; \mathrm{M}-\mathrm{N}: 1 \mu \mathrm{m}$.

formados hasta por ocho poroides redondeados (Fig. 3K, M). Valvocópula ancha, con fimbrias desarrolladas en la parte interior (Fig. 3L).
Description: Frustules in girdle view rectangular. Valves cruciform, isopolar to slightly heteropolar, rostrate with rounded apices of variable length. 
Axial area narrow, lanceolate, delimited by slightly radial striae. Striae uniseriated, formed by apically elongated areolae, appearing sunken into the valve and uninterrupted from valve face to mantle. Striae decrease in width toward valve margin and toward valve center. Spines solid, marginal, located between striae, also present at the poles, with abruptly spatulate apices. Volae were not observed and are probably reduced due to small areolar aperture. APF present at both apices, unequally developed, formed by up to 8 poroids. Valvocopula wide with well-developed fimbriae in the pars interior.

Dimensiones: eje apical 11,6-15,3 $\mu \mathrm{m}(\mathrm{N}=41)$; eje transapical 4-5,5 $\mu \mathrm{m}(\mathrm{N}=41)$; relación largo/ ancho: 2,4-3,3. Largo del ápice: 2,5-3,5 $\mu \mathrm{m}(\mathrm{N}=20)$. Estrías 9-12 en $10 \mu \mathrm{m}(\mathrm{N}=41)$.

Holotipo: ARGENTINA. Prov. Catamarca: Dpto. Antofagasta, 20-01-2005, Seeligmann 261051 (LIL). Parcialmente ilustrado en Fig. 3A-G.

Localidad tipo: Laguna Salitre 26 14 '51's 66 54'04'W, $4248 \mathrm{~m}$ snm (Dpto. Antofagasta, Catamarca, Argentina).

Etimología: el epíteto de la especie se refiere a que fue encontrada en las inmediaciones del volcán Galán.

Observaciones: Las características ultraestructurales (tipo de estrías, fimbrias bien desarrolladas y valvocópula ancha) muestran claramente que esta especie pertenece a Staurosirella, aunque es la única que tiene CPAs pequeños. Las valvas más pequeñas de $S$. rhomboides Morales \& Manoylov (Morales \& Manoylov, 2006a) se parecen a $S$. galanensis sp. nov., sin embargo, la forma de la valva es romboide en $S$. rhomboides y sus dimensiones son mayores, alcanzando longitudes de hasta $55 \mu \mathrm{m}$, un ancho valvar entre 5.5-9 $\mu \mathrm{m}$ y una densidad de estrías menor( $6,5-9 \mu \mathrm{m}$ en $10 \mu \mathrm{m})$, medidas que no se superponen con los de la nueva especie. Los CPAs son también mucho menos desarrollados en $S$. galanensis, en la que no se observa la marcada heteropolaridad de S. rhomboides (Morales \& Manoylov, 2006a, fig. 96).

Staurosirella galanensis sp. nov. es también diferente a la otra especie cruciforme del género, $S$. leptostauron (Ehrenberg) Williams \& Round. Si bien las formas más pequeñas pueden superponerse en su longitud (12-33 $\mu \mathrm{m})$ a las de $S$. galanensis sp. nov., las valvas de $S$. leptostauron tienen una construcción mucho más robusta y el ensanchamiento de las valvas en el área central es mucho más pronunciado, llegando a tener incluso formas cuneadas a ambos lados de la valva. Asimismo, en S. leptostauron las estrías son mucho más anchas y marcadas y las valvas son también más anchas (7-19 $\mu \mathrm{m})$ (Morales \& Manoylov, 2006a, figs. 1-12). S. acidophila Almeida, Wetzel, Morales, Ector \& Bicudo también tiene valvas romboidales, sin embargo la forma es lanceolada, con ápices relativamente más estrechos (Almeida et al., 2015, figs. 2-31). Además, las valvas llegan a ser más largas (hasta 19,5 $\mu \mathrm{m}$ ), más anchas (hasta $6,8 \mu \mathrm{m}$ ) y la densidad de estrías es mucho menor (8-9 en $10 \mu \mathrm{m})$, los CPAs son mucho más desarrollados (Almeida et al., 2015, figs. 33, 34,37 ) y las espinas son mucho más desarrolladas, pudiendo encontrarse una o dos filas de tres espinas sobre una misma interestría (Almeida et al., 2015, figs. 33, 34, 37, 38).

Staurosirella salitrensis Seeligmann, C. T., N. I. Maidana \& E. A. Morales sp. nov. (Fig. 4A-O)

Valvas marcadamente heteropolares. El extremo apical desde rostrado hasta no diferenciado del cuerpo principal, con el ápice redondeado, de ancho variable, generalmente tiene un escalón más o menos evidente (Fig. 4H-I), con o sin estrías y con un CPA reducido, con escasos poroides, difíciles de observar ya que se ubican en el manto valvar (Fig. 4M). El extremo basal es más angosto, redondeado $\mathrm{y}$, en algunos ejemplares, rostrado (Fig. 4A-G); con un CPA bien desarrollado, ubicado algo más arriba de la unión manto-superficie valvar y que se extiende hasta el extremo abvalvar del manto (Fig. 4I, K, N). Área axial angosta, lineal a lanceolada. Estrías anchas, dispuestas en forma alterna respecto del eje apical, no visibles en el extremo basal. La superficie valvar es ondulada, con costillas marcadas separando las estrías, que se ubican en depresiones (Fig. 4L, O). Las estrías están formadas por aréolas alargadas en sentido apical. En cada estría las aréolas de los extremos son mucho más cortas que las centrales (Fig. 4H). Las estrías son normalmente uniseriadas aunque, a veces, las areolas aparecen divididas en dos o en tres aréolas más pequeñas (Fig. 4I, L, O), a través de conexiones silíceas (cross-bars). Estas divisiones, pueden verse en un mismo individuo en algunas, todas o ninguna de las estrías (Fig. 4L, O). Las espinas, cuando presentes, son muy pequeñas, sólidas y cónicas, 


\section{T. Seeligman et al. - Fragilariaceae en humedales de altura}
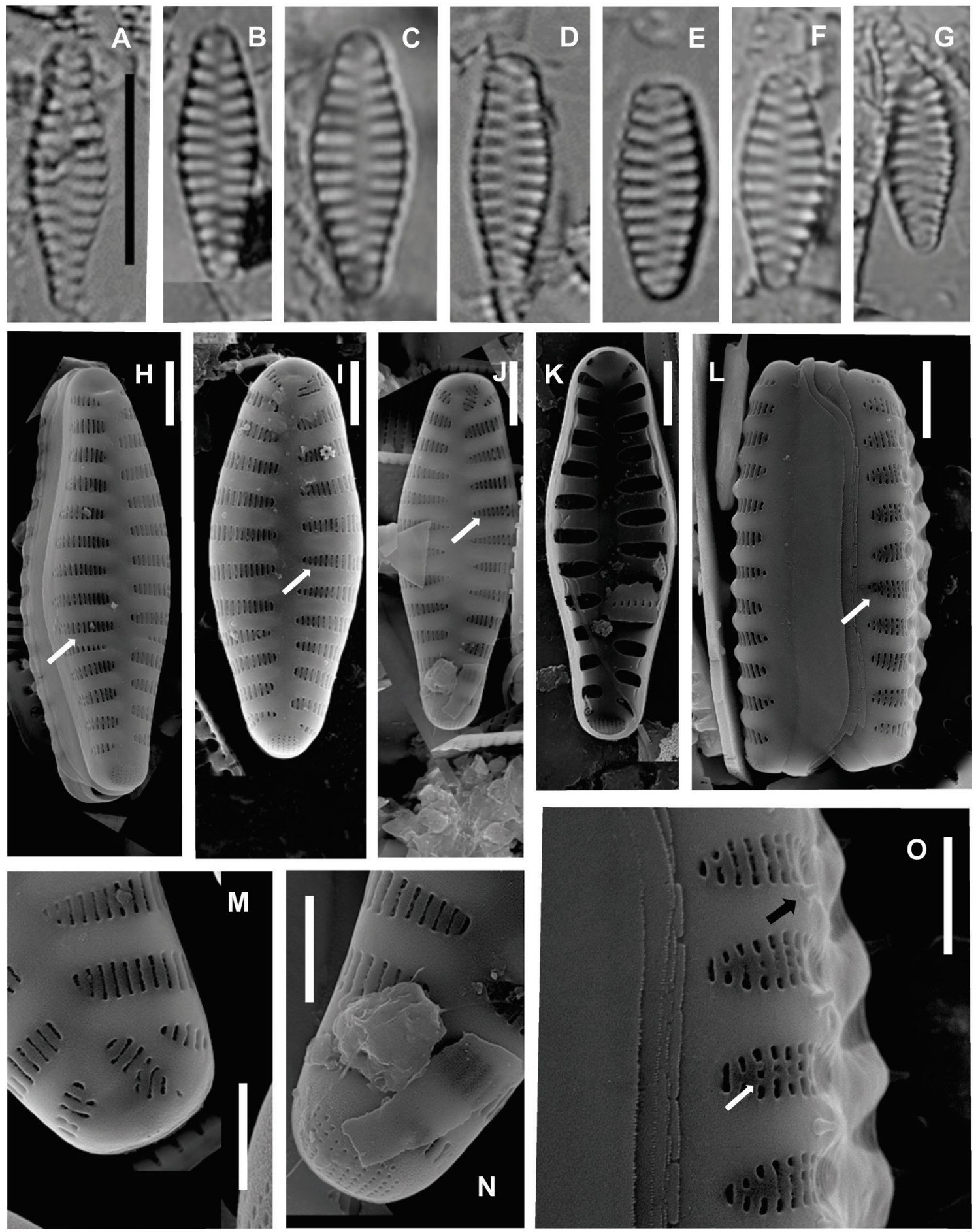

Fig. 4. Staurosirella salitrensis. A-G: (MO). H-O: (MEB). H-J: vistas externas con (H-I) y sin (J) escalones en el extremo apical y CPA en el extremo basal de la valva. K: vista interna. L, O: vista cingular donde se observa la superficie valvar ondulada, las estrías están localizadas en una sinuosidad y cada espina cónica está ubicada en una depresión circular (flecha negra). M: detalle de uno de los ápices con escalón. N: CPA bien desarrollado. Las flechas blancas señalan la formación de estrías seriadas. Escalas = A-G: $10 \mu \mathrm{m} ; \mathrm{H}-\mathrm{L}: 2 \mu \mathrm{m} ; \mathrm{M}-\mathrm{O}: 1 \mu \mathrm{m}$. 
ubicadas en depresiones circulares, sobre las costillas (Fig. 4O). Cíngulo compuesto por varias bandas no perforadas, siendo la valvocópula la más ancha. No se pudo determinar el carácter abierto o cerrado de los elementos del cíngulo. (Fig. 4L).

Description: Valves clearly heteropolar. Apical end varying from rostrate to non-differentiated from the rest of the valve, generally with a depression (step), with or without striae and with a reduced apical pore field composed of small poroids, difficult to observe since they are situated on the valve mantle. Basal apex is narrower than apical one, rounded to rostrate always with a well-developed apical pore field, located at the transition between valve face and mantle and extending to the abvalvar side of the latter. Axial area narrow, linear to lanceolate. Striae wide, alternate, not visible toward basal end. Valve surface undulate with marked costae, separating striae located on depressions. The striae formed by apically elongated areolae. In each stria, areolae at ends are shorter than middle ones. Striae are usually uniseriate although, sometimes, the areolas are subdivided into 2 or 3 shorter ones through siliceous connections (cross-bars). Within the same population, some individuals lack such subdivisions while others have partial or totally subdivided striae, thus resembling members of Punctastriata. Spines, when present, small, solid, conical and located on circular depressions on the costae. Cingulum composed of several non-perforated bands, the valvocopula being the widest.

Dimensiones: eje apical 9,8-13,5 $\mu \mathrm{m}(\mathrm{N}=30)$; eje transapical 3,2-4,4 $\mu \mathrm{m}(\mathrm{N}=30)$. Estrías $10-14$ en 10 $\mu \mathrm{m}(\mathrm{N}=30)$; aréolas 58-66 en $10 \mu \mathrm{m}$.

Holotipo: ARGENTINA. Prov. Catamarca: Dpto. Antofagasta, 20-01-2005, Seeligmann 261051 (LIL). Parcialmente ilustrado en Fig. 4A-G.

Localidad tipo: Laguna Salitre $26^{\circ} 14$ '51' S 66 54'04'W, $4248 \mathrm{~m}$ snm (Dpto. Antofagasta, Catamarca, Argentina).

Etimología: el epíteto de la especie se refiere a que fue encontrada en la laguna Salitre.

Observaciones: Si bien al MEB las valvas del material estudiado guardan cierta semejanza con S. martyi (Héribaud) Morales \& Manoylov (ver Morales \& Manoylov, 2006a, Figs. 105, 106); al MO lucen mucho menos silicificadas, menos ovaladas y tienen mayor densidad de estrías (comparar sus Figs. 34-43 con nuestras Fig. 4A-G).
S. martyi nunca tiene espinas y solo tienen CPAs en el polo basal (Morales \& Manoylov, 2006a, figs. 102-104) mientras que en $S$. salitrensis sp. nov. están en ambos polos, aunque el del polo apical es mucho más reducido que el del basal. Asimismo, hay una diferencia en las bandas cingulares, las de $S$. martyi son notablemente convexas en su margen abvalvar (Morales \& Manoylov, 2006a, fig. 107) mientras que en $S$. salitrensis sp. nov. este margen es paralelo al borde abvalvar del manto. S. incognita Morales \& Manoylov también es heteropolar aunque con el polo basal mucho más delgado y cuneado; con el escalón siempre presente en el polo apical (Morales \& Manyolov, 2006b, figs. 7-26). Las valvas de $S$. incognita llegan a ser más largas (hasta $21 \mu \mathrm{m}$ ), son más anchas $(4-6 \mu \mathrm{m})$, las estrías son mucho menos densas (7-9 in $10 \mu \mathrm{m})$ y los CPAs son mucho más desarrollados (Morales \& Manyolov, 2006b, figs. 24, 25). Si bien las dimensiones de $S$. salitrensis se superponen con las de $S$. pinnata sensu Morales (Morales, 2010), la heteropolaridad en esta última no es tan notoria y las valvas tienden a ser más elípticas y no ovaladas como en $S$. salitrensis. Además, las espinas están bien desarrolladas en $S$. pinnata.

La presencia de un escalón en el polo apical también fue observada para otras especies del género, como S. martyi, S. confusa (Morales, 2005) y S. incognita (Morales \& Manoylov, 2006b).

La presencia de divisiones en las aréolas en el género Staurosirella ya ha sido mostrada para varias especies (Morales, 2005; Morales et al., 2014b).

Pseudostaurosira altiplanensis Lange-Bertalot \& U. Rumrich in Rumrich et al. 2000 (Fig 5A-B)

Valvas estrictamente elípticas, con extremos truncos redondeados, nunca rostrados.

Dimensiones: eje apical $6,8 \mu \mathrm{m}$, eje transapical 2,5-3 $\mu \mathrm{m}$. Estrías 12-15 en $10 \mu \mathrm{m}$.

Material estudiado:laguna Salitre.

Pseudostaurosira altiplanensis es citada por primera vez para Argentina. Fue encontrada por Rumrich et al. (2000) en el Parque Nacional Lauca (4.300 m snm, Chile).

Pseudostaurosira ferrarioae Seeligmann, C. T., N. I. Maidana \& E. A. Morales sp. nov. (Fig. 6A-M)

Valvas rómbico-lanceoladas, con ápices alargados, subrostrados, raramente subcapitados, redondeados. Área axial ancha, lanceolada. Estrías 


\section{T. Seeligman et al. - Fragilariaceae en humedales de altura}
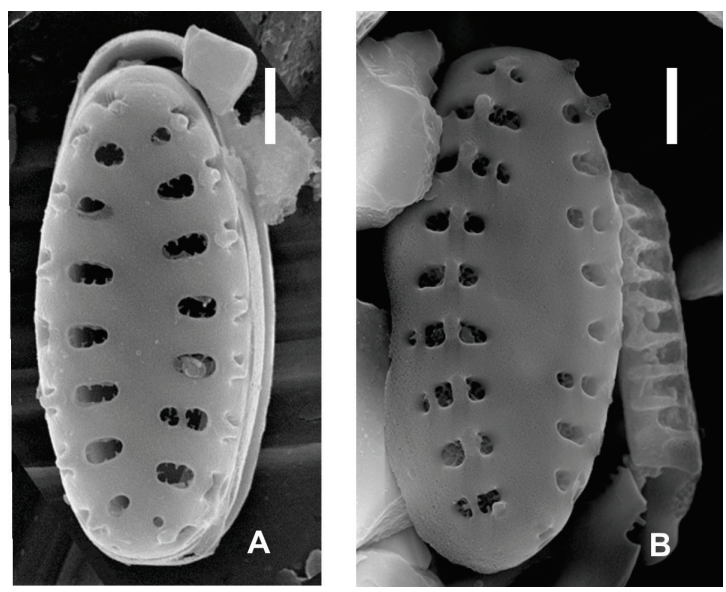

Fig. 5. Pseudostaurosira altiplanensis. A-B: (MEB) vistas externas. Escalas= A-B: $1 \mu \mathrm{m}$. cortas, radiales, formadas por dos, raramente tres, aréolas grandes, alargadas en sentido transapical (Fig. 6I). Una o dos aréolas en la superficie valvar y una en el manto, ocluidas por volae dicotómicamente ramificadas (Fig. 6M).

CPAs desarrollados (Fig. 6I-L), formados por 6-8 filas de pequeños poroides redondeados. Espinas, cuando presentes, muy cortas y cónicas, interrumpiendo las estrías (Fig. 6I-J).

Description: Valves rhomboid with elongated, subrostrate, rarely subcapitate apices. Striae formed by two, rarely three large, elongated areolae. One or two areolae on valve face and one on valve mantle; occluded by dichotomously branched volae. Apical pore fields (APF) developed, formed by 6-8 rows of small, rounded
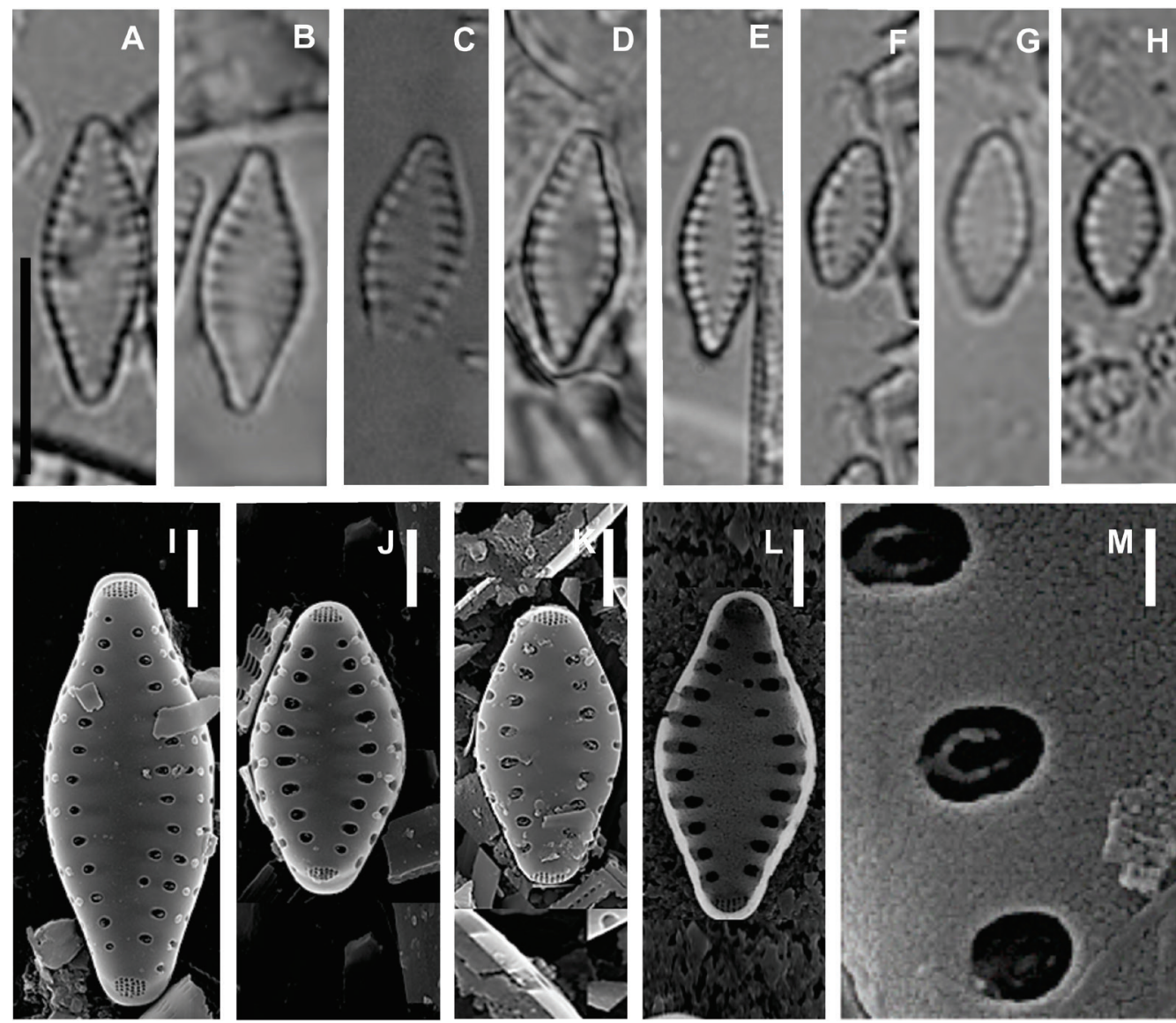

Fig. 6. Pseudostaurosira ferrarioae. A-H: (MO). I-M: (MEB). I-K: vistas externas de las valvas mostrando estrías formadas por 1-2 aréolas, espinas cortas y CPAs formados por pequeños poroides. L: vista interna. M: detalle de la vista externa mostrando aréolas ocluidas por volae dicotómicamente ramificadas. Escalas $=\mathrm{A}-\mathrm{H}: 10 \mu \mathrm{m} ; \mathrm{I}-\mathrm{L}: 2 \mu \mathrm{m} ; \mathrm{M}: 200 \mathrm{~nm}$. 
poroids. Spines, when present, short and conical, interrupting the striae.

Dimensiones: eje apical 8,5-10 $\mu \mathrm{m}(\mathrm{N}=37)$, eje transapical 3,8-4,4 $\mu \mathrm{m}(\mathrm{N}=37)$. Estrías, $13-15$ en 10 $\mu \mathrm{m}(\mathrm{N}=37)$.

Holotipo: ARGENTINA. Prov. Catamarca: Dpto. Antofagasta, 20-01-2005, Seeligmann 26105- 2 (LIL). Parcialmente ilustrado en Fig. 6A-H.

Localidad tipo: Laguna Salitre 26 14 '51's 66 54'04”'W, $4248 \mathrm{~m}$ snm (Dpto. Antofagasta, Catamarca, Argentina).

Etimología: dedicamos esta nueva especie a la Dra. Martha Ferrario, maestra de diatomólogos.

Observaciones: Si bien la mayoría de las especies de Pseudostaurosira tiene CPAs reducidos (CejudoFigueiras et al., 2011), hay taxones, como $P$. parasitica (W. Smith) Morales y la nueva especie aquí propuesta, que los tienen bien desarrollados (ver Morales et al., 2015, figs. 95-96). Sin embargo, en $P$. parasitica el CPA está hundido en el ápice valvar, lo cual se observa también en vista interna, como un adelgazamiento de toda la placa del CPA (Morales, 2003, figs. 54-58, 64, Morales et al., 2015, figs. 95-98).

Al MO, la nueva especie es muy parecida a P. brevistriata (Grunow) Williams \& Round y a P. microstriata (Marciniak) Flower. Si bien $P$. brevistriata y $P$. ferrarioae sp. nov. poseen aproximadamente el mismo ancho valvar y la misma densidad de estrías, las valvas de $P$. ferrarioae sp. nov. son más cortas (8,5-10 $\mu \mathrm{m}$ contra 11-19 $\mu \mathrm{m}$ en $P$. brevistriata). En P. brevistriata las valvas son mucho más lanceoladas y las espinas son robustas (Morales et al., 2015, figs. 107-143). Los CPAs de $P$. brevistriata también están hundidos (Morales et al., 2015, figs. 140-142) pero están muy reducidos (4 poroides) mientras que en P. ferrarioae sp. nov. están bien desarrollados, con numerosos poroides dispuestos regularmente en hileras (Fig. 6 I-J). P. microstriata tiene valvas mucho más pequeñas (5,5-7,5 de largo y 2,1-3,3 de ancho) y la densidad de estrías es también mucho mayor en esta especie (18-22 en $10 \mu \mathrm{m})$. El contorno de P. microstriata es menos romboidal y más lanceolado, los extremos incluso llegan a ser subrostrados y los ápices son más redondeados que los de $P$. ferrarioae $\mathrm{sp}$. nov. (Marciniak, 1982). Adicionalmente, los CPAs de $P$. microstriata son muy reducidos y se circunscriben a no más de cuatro poros. Pseudostaurosira microstriata var. spinosa Flower difiere de la variedad nominal por la menor densidad de estrías (17 en $10 \mu \mathrm{m})$, la presencia de espinas bien desarrolladas, ubicadas sobre las estrías y CPAs reducidos (Flower, 2005), contrariamente a lo que sucede en $P$. ferrarioae sp. nov.

Pseudostaurosira ferrarioae sp. nov. también se asemeja a $P$. elliptica pero esta última es más angosta $(3-3,5 \mu \mathrm{m})$, posee espinas bien desarrolladas interrumpiendo sus estrías, CPAs con espínulas y hundidos interna y externamente (Edlund et al., 2006, figs. 17-24).

Los CPAs de $P$. ferrarioae sp. nov. se asemejan a los de P. moralesi Graeff, Kociolek \& S.R. Rushforth en su grado de desarrollo pero nuestro material carece de las estrías fantasma que caracterizan a $P$. moralesi. También, la forma valvar es elíptica con ápices cuneados en $P$. moralesi y los CPAs están hundidos hacia el interior (Graef et al., 2013 figs. 56-63).

Pseudostaurosira ferrarioae también difiere de $P$. decipiens en que esta última posee valvas lanceoladas, más anchas $(4-6 \mu \mathrm{m})$ y los CPAs se hallan hundidos en ambos ápices valvares tanto externa como internamente (Morales et al., 2012, figs. 39, 42, 44). Además, $P$. decipiens también posee las alas o discos flotantes en la parte interna y externa de las aperturas areolares (Morales et al., 2012, figs. 39, 40, 44), los cuales no se han observado en $P$. ferrarioae.

La nueva especie también se diferencia de $P$. laucensis por la forma de la valva, que en el caso de esta última es lanceolada con ápices muy delgados en relación al resto de la valva; las valvas pueden ser más largas (hasta $20 \mu \mathrm{m}$ ) y más anchas (hasta $5,5 \mu \mathrm{m}$ ). Las estrías de $P$. laucensis están más desarrolladas, delimitando un área axial menos ancha que la de P. ferrarioae (Rumrich, et al., 2000, figs. 10-20, $22,23)$. Estas últimas figuras muestran también las estrías fantasma de $P$. laucensis que están ausentes en $P$. ferrarioae. Adicionalmente, las espinas de $P$. laucensis están reducidas a grupos de prominencias que se hallan interrumpiendo las estrías y que, en algunos casos, parecen formar un ribete continuo a lo largo del borde de la cara valvar, próximo a su unión con el manto (Rumrich et al., 2000, lám. 3, fig. 22). Si bien los CPAs están desarrollados en P. laucensis (Rumrich et al., 2000, lám. 3, figs 22, 23), estos se hallan hundidos en el ápice valvar en vista externa. No existen imágenes de MEB de la vista interna de este taxon.

Algunos ejemplares de P. tenuis Morales \& Edlund tienen cierta similitud con $P$. ferrarioae (Morales $\&$ Edlund, 2003, Fig. 22) pero ambas especies se diferencian por el ancho valvar $(2 \mu \mathrm{m})$ y el número de estrías $(23$ en $10 \mu \mathrm{m})$. 


\section{T. Seeligman et al. - Fragilariaceae en humedales de altura}

Pseudostaurosira aff. polonica (M. Witak \& LangeBertalot) E. A. Morales \& M. B. Edlund (Fig. 7A-G)

Valvas anchamente elípticas, con extremos no rostrados y ápices ligeramente aguzados en especímenes pequeños y redondeados en los más grandes.

Dimensiones: eje apical 7,8-20,3 $\mu \mathrm{m}$, eje transapical 2,5-3 $\mu \mathrm{m}$. Estrías 13-15 en $10 \mu \mathrm{m}$.

Material estudiado: vega de la laguna Negra.

Observaciones: Nuestros ejemplares se asemejan a Pseudostaurosira polonica por la forma, tamaño, tipo de espinas y aréolas con volae desarrolladas y se diferencian porque los CPAs están muy poco desarrollados o están formados por un poroide. P. polonica fue reportada para estuarios en Polonia (Witkowski et al., 1995) y para agua dulce en Mongolia (Morales \& Edlund, 2003). Esta discontinuidad geográfica y las preferencias ecológicas aparentemente diferentes sugieren que " $P$. polonica" podría tratarse de un complejo de especies en el cual nuestro material podría estar incluido.

\section{Conclusiones}

Aunque existen numerosos estudios realizados dentro de este grupo de diatomeas, la falta de coherencia en la taxonomía y en la información brindada por la literatura (Morales et al., 2010) no permite aún establecer las preferencias ecológicas de la mayoría de los taxones hasta ahora descritos. Para algunos autores las Fragilariaceae son frecuentes en alta montaña, donde estos organismos deben adaptarse a bajas temperaturas, con prolongados períodos con hielo, cambios bruscos de las condiciones físicoquímicas, bajas concentraciones de nutrientes y alta UV (Schmidt et al., 2004a). Contribuciones realizadas para ambientes de alta montaña por otros autores (Álvarez-Blanco et al., 2011; Morales \& Edlund, 2003; Morales et al., 2010, 2012; Rumrich et al., 2000; Schmidt et al., 2004a; 2004b, entre otros) indican que Staurosira es el género encontrado con mayor frecuencia en estos ambientes, seguido de Pseudostaurosira, siendo Staurosirella el menos frecuente. Sin embargo, debe considerarse que los autores mencionados tienen conceptos taxonómicos diferentes para la circunscripción de estos géneros.

El hallazgo de tres especies nuevas en muestras provenientes de sólo tres humedales sugiere que son necesarios nuevos estudios para conocer la real diversidad de las diatomeas fragilarioides en alta montaña y clarificar sus preferencias ecológicas.
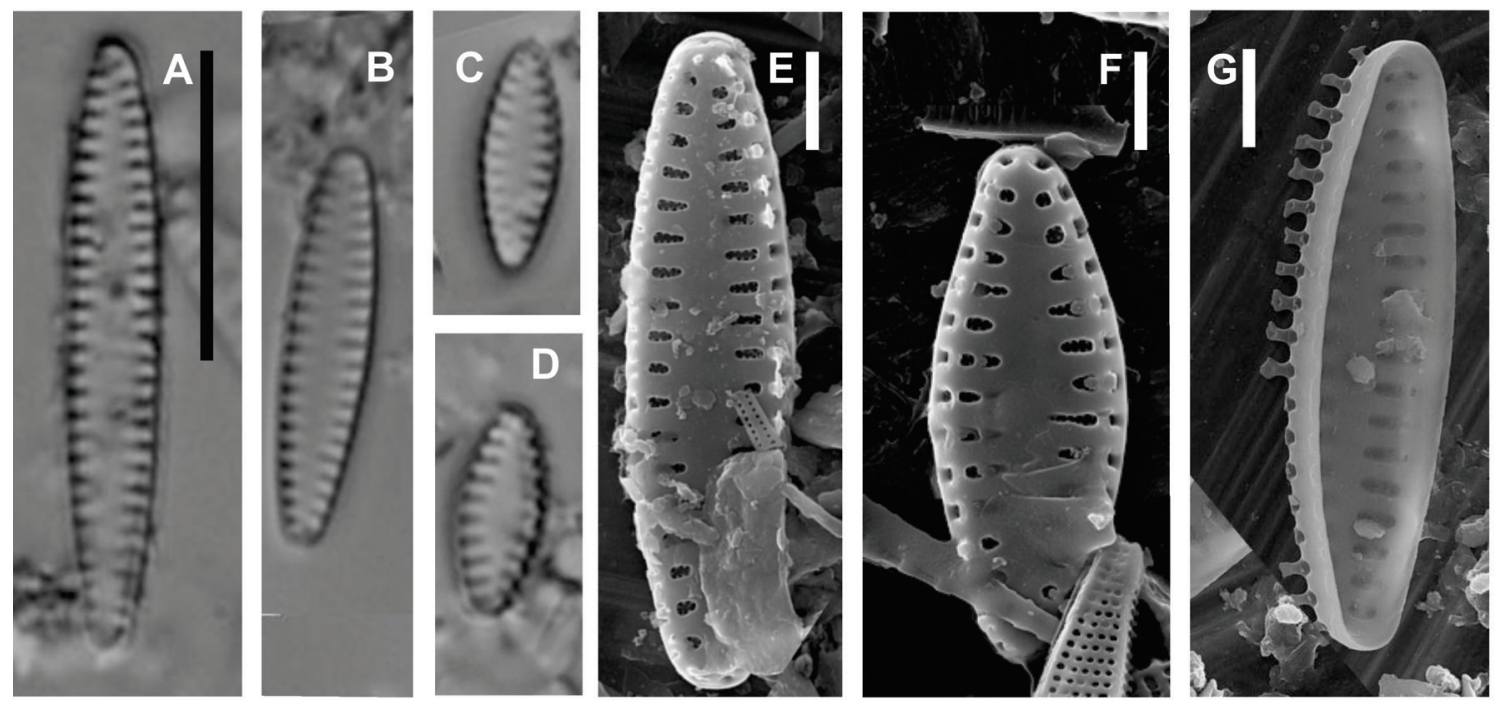

Fig. 7. Pseudostaurosira aff. polonica. A-D: (MO). E-G: (MEB). E-F: vistas externas. E: espinas espatuladas interrumpiendo las estrías. G: vista interna. Escalas = A-D: $10 \mu \mathrm{m}$; E-G: $2 \mu \mathrm{m}$. 


\section{Agradecimientos}

Agradecemos a la Fundación Miguel Lillo por el lugar de trabajo proporcionado. Este trabajo fue cofinanciado por la Unión Europea a través del Fondo de Desarrollo Regional Europeo en el marco de COMPETE 2020 (Operational Programme Competitiveness and Internationalization) y a través del Proyecto ICT (UID/GEO/04683/2013) con referencia POCI-01-0145-FEDER-007690.

\section{Bibliografía}

ALMEIDA, P. D., C. E. WETZEL, E. A. MORALES, L. ECTOR \& D. C. BICUDO. 2015. Staurosirella acidophila sp. nov., a new araphid diatom (Bacillariophyta) from southeastern Brazil: ultrastructure, distribution and autecology. Cryptogamie Algol. 36: 255-270.

ÁLVAREZ-BLANCO, I., C. CEJUDO-FIGUEIRAS, I. de GODOS, R. MUÑOZ \& S. BLANCO. 2011. Las diatomeas de los salares del Altiplano boliviano: singularidades florísticas. Bol. R. Soc. Esp. Hist. Nat. Secc. Biol. 105: 67-82.

BARBER, H. G. \& E. Y. HAWORTH. 1981. A guide to the morphology of the diatom frustule with a key to the British freshwater genera. Freshwater Biol. Assoc. Scient. Publ. 44: 1-112.

BATTARBEE, E. W. 1986. Diatom Analysis. In: BERGLUND, B. E. (ed.), Handbook of Holocene Palaeoecology and Palaeohydrology, pp. 527-570. J. Wiley \& Sons, New York.

CAZIANI, S. \& E. DERLINDATI. 2000. Abundance and habitat of Andes flamingos in northwestern Argentina. Waterbirds 23 (Special publication 1): 121-133.

CEJUDO-FIGUEIRAS, C., E. A. MORALES, C. E. WETZEL, S. L. BLANCO, L. HOFFMANN \& L. ECTOR. 2011. Analysis of the type of Fragilaria construens var. subsalina (Bacillariophyceae) and description of two morphologically related taxa from Europe and the United States. Phycologia 50: 67-77.

EDLUND, M. B., E. A. MORALES \& S. A. SPAULDING. 2006. The type and taxonomy of Fragilaria elliptica Schumann, a widely misconstrued taxon. In: WIRTOWSKI, A. (ed.), Eighteenth International Diatom Symposium 2004, pp. 53-59. Biopress Limited, Bristol.

FLOWER, R. J. 2005. A taxonomic and ecological study of diatoms from freshwater habitats in the Falkland Islands, South Atlantic. Diatom Res. 20: 23-96.

GONZÁLEZ ACHEM, A. L., C. T. SEELIGMANN \& M. ALDERETE. 2014. Variaciones espacio-temporales de la flora diatomológica en Laguna de Los Pozuelos (Jujuy, Argentina). Bol. Soc. Argent. Bot. 49: 177-193.
GRAEFF, C. L., J. P. KOCIOLEK \& S. R. RUSHFORTH. 2013. New and interesting diatoms (Bacillariophyta) from Blue Lake warm springs, Tooele County, Utah. Phytotaxa 153: 1-38.

MAIDANA, N. I. \& C. T. SEELIGMANN. 2006. Diatomeas (Bacillariophyceae) de ambientes acuáticos de altura de la Provincia de Catamarca, Argentina II. Bol. Soc. Argent. Bot. 41: 1-13.

MAIDANA, N. I. \& C. T. SEELIGMANN. 2014. Phytoplankton in high mountain wetlands of Argentina. In: TELL, G, I. IZAGUIRRE \& I. O'FARRELL, (eds.), Freshwater Phytoplankton of Argentina. Advance in Limnology 65, pp. 23-35. Schweizerbart Science Publishers. Stuttgart.

MAIDANA, N. I. \& C. T. SEELIGMANN. 2015. Diatomeas (Bacillariophyceae) en humedales de altura de la provincia de Catamarca (Argentina). III. Bol. Soc. Argent. Bot. 50: 447-466.

MAIDANA, N. I., C. T. SEELIGMANN \& M. MORALES. 2011. El género Navicula sensu stricto (Bacillariophyceae) en humedales de altura de Jujuy, Argentina. Bol. Soc. Argent. Bot. 46: 13-29.

MARCINIAK, B. 1982. Late glacial and Holocene new diatoms from glacial Lake Przedni Staw in the Przedni Stawów Polskich Valley, Polissh Tatra Mts. Acta Geol. Acad. Sci. Hung. 25: 161-71.

MORALES, E. A. 2001. Morphological studies in selected fragilarioid diatoms (Bacillariophyceae) from Connecticut waters (USA). Proc. Acad. Nat. Sci. Phila. 151: 105-120.

MORALES, E. A. 2002. Studies in selected fragilarioid diatoms of potential indicator value from Florida (USA) with notes on the genus Opephora Petit (Bacillariophyceae). Limnologica 32: 102-113.

MORALES, E. A. 2003. On the taxonomic status of the genera Belonastrum and Synedrella proposed by Round and Maidana (2001). Crypotogamie Algol. 24: 277-288.

MORALES, E. A. 2005. Observations of the morphology of some known and new fragilarioid diatoms (Bacillariophyceae) from rivers in the USA. Phycol. Res. 53: 113-133.

MORALES, E A. 2010. Citing online sources: Staurosirella pinnata. In Diatoms of North America. [online]. Disponible en: https://diatoms. org/species/staurosirella_pinnata. htm [Acceso: 03 July 2018].

MORALES, E. A. \& M. B. EDLUND. 2003. Studies in selected fragilarioid diatoms (Bacillariophyceae) from Lake Hovsgol, Mongolia. Phycol. Res. 51: 225-239.

MORALES, E. A. \& K. M. MANOYLOV. 2006a. Morphological studies on selected taxa in the genus Staurosirella Williams et Round (Bacillariophyceae) from rivers in North America. Diatom Res. 21: 343-364. 


\section{T. Seeligman et al. - Fragilariaceae en humedales de altura}

MORALES, E. A. \& K. M. MANOYLOV. 2006 b. Staurosirella incognita Morales et Manoylov sp. nov., a non-spiny species from North America, with an emended description of Staurosirella Williams et Round (Bacillariophyceae). In: WITKOWSKI, A. (ed.), Proceedings of the Eighteenth International Diatom Symposium, pp. 325-336. Biopress Limited, Bristol.

MORALES, E. A., E. FERNÁNDEZ \& P. J. KOCIOLEK. 2009. Epilithic diatoms (Bacillariophyta) from cloud forest and alpine streams in Bolivia, South America 3: diatoms from Sehuencas, Carrasco National Park, Department of Cochabamba. Acta Bot. Croat. 68: 263-283.

MORALES, E. A., M. B. EDLUND \&. S. A SPAULDING. 2010. Description and ultrastructure of araphid diatom species (Bacillariophyceae) morphologically similar to Pseudostaurosira elliptica (Schumann) Edlund et al. Phycol. Res. 58: 97-107.

MORALES, E. A., M. H. NOVAIS, G. CHÁVEZ, L. HOFFMANN \& L. ECTOR. 2012. Diatoms (Bacillariophyceae) from the Bolivian Altiplano: three new araphid species from the Desagüadero River draining Lake Titicaca. Fottea 12: 41-58.

MORALES, E. A, C. E. WETZEL, S. F. RIVERA, B. VAN DE VIJVER \& L. ECTOR. 2014a. Current taxonomic studies on the diatom flora (Bacillariophyceae) of the Bolivian Altiplano, South America, with possible consequences for palaeoecological assessments. J. Micropalaeontol. 33: 121-129.

MORALES, E. A., K. BUCZKÓ, C. E. WETZEL, M. H. NOVAIS, N. OGNJANOVA-RUMENOVA, L. HOFFMANN \& L. ECTOR. 2014b. Transfer of Staurosira grunowii to Staurosirella. Diatom Res. 29: 105-110.

MORALES, E. A., C. E. WETZEL, B. VAN DE VIJVER \& L. ECTOR. 2015. Morphological studies on type material of widely cited araphid diatoms (Bacillariophyta). Phycologia 54: 455-470.

MORRONE, J. J. 2002. Presentación sintética de un nuevo esquema biogeográfico de América Latina y el Caribe. In: COSTA, C., A. VANIN, J. M. LOBO \& A. MELIC (eds.), Red Latinoamericana de Biogeografía y Entomología sistemática. Monografías Tercer Milenio 2, pp. 267-275. SEA, Zaragoza.
ROSS, R., E. J. COX, N. I. KARAYEVA, D. G. MANN, T. B. B. PADDOCK, R. SIMONSEN \& P. A. SIMS. 1979. An amended terminology for the siliceous components of the diatom cell. Nova Hedwigia, Beih. 64: 513-533.

ROUND, F. E., R. M. CRAWFORD, \& D. G. MANN. 1990. The Diatoms. Biology and Morphology of the Genera. Cambridge University Press, Cambridge.

RUMRICH, U., H. LANGE-BERTALOT \& M. RUMRICH. 2000. Diatoms of the Andes: from Venezuela to Patagonia/Tierra del Fuego. Iconogr. Diatomol. 9: 1-649.

SCHMIDT, R., C. KAMENIK, H. LANGE-BERTALOT \& R. KLEE. 2004a. Fragilaria and Staurosira (Bacillariophyceae) from sediment surfaces of 40 lakes in the Austrian Alps in relation to environmental variables, and their potential for palaeoclimatology. J. Limnol. 63: 171-189.

SCHMIDT, R., H. LANGE-BERTALOT, \& R. KLEE. 2004b. Staurosira parasitoides sp nova and Staurosira microstriata (Marciniak) Lange-Bertalot from surface sediment samples of Austrian alpine lakes. Algol. Stud. 114: 1-9.

SEDDON, A. W. R., A. WITKOWSKI, C. A. FROYD, J. K. KURZYDŁOWSKI, J. GRZONKA \& K. J. WILLIS. 2104. Diatoms from isolated islands II: Pseudostaurosira diablarum, a new species from a mangrove ecosystem in the Galápagos Islands. Diatom Res. 29: 201-211.

SEELIGMANN, C. T. \& N. I. MAIDANA. 2003. Diatomeas (Bacillariophyceae) en ambientes de altura de la Provincia de Catamarca (Argentina). Bol. Soc. Argent. Bot. 38: 39-50.

SEELIGMANN, C. T., N. I. MAIDANA \& M. MORALES. 2008. Diatomeas (Bacillariophyceae) de humedales de altura de la Provincia de Jujuy Argentina. Bol. Soc. Argent. Bot. 43: 1-17.

WILLIAMS, D. M. \& F. ROUND. 1987. Revision of the genus Fragilaria Lyngbye. Diatom Res. 2: 267-288.

WITKOWSKI, A., H. LANGE-BERTALOT \& M. WITAK. 1995. Diatom taxa of unusual frustule structure belonging to the genus Fragilaria. Frag. Florist. Geobot. 40: 729-741.

Recibido el 11 de abril de 2018, aceptado el 7 de septiembre de 2018. Editora: Eugenia Alicia Sar. 
